\title{
PERFORMANCE ANALYSIS OF FULLY DEPLETED DUAL MATERIAL GATE (DMG) SOI MOSFET AT 25NM TECHNOLOGY
}

\author{
B.H.V Shrikant ${ }^{1}$, Ashwani K. Rana ${ }^{2}$ \\ ${ }^{1}$ M.Tech Scholar, Electronics \& Communication Engineering Department, NIT Hamirpur, H.P, India \\ ${ }^{2}$ Assistant Professor, Electronics \& Communication Engineering Department, NIT Hamirpur, H.P, India
}

\begin{abstract}
The emergence of CMOS technology in the semiconductor industry is predominant. However, there is degradation of MOSFET characteristics as the technology is scaled down to nanometer regime. In this paper, Dual Material Gate MOSFET is analyzed to study the performance characteristics like DIBL, Subthreshold Slope and $I_{\text {on }} / I_{\text {off }}$ ratio using TCAD simulation. We observed that the DMG MOSFET is effective in reducing DIBL. Also, by using $\mathrm{HfO}_{2}$ as spacer, we obtained improvement in the $\mathrm{I}_{\text {on }} / I_{\text {off }}$ ratio and reduction in DIBL and Subthreshold Slope.
\end{abstract}

Keywords: DIBL, Silicon-On-Insulator, Single Material Gate, Dual Material Gate, MOSFET, SCE, TCAD, Ion/Ioff

\section{INTRODUCTION}

The Dual Material Gate is implemented by making half of the gate with one metal and the other half with another metal. There is a difference in the workfunction of both the metals such that for an n-channel MOSFET, the workfunction of the metal used in the source side is higher than the workfunction of the metal used in the drain side and vice versa for a $p$ channel MOSFET, which in turn introduces a step in the channel potential. This paper is a study of performance improvements of DMG MOSFET and its effective suppression of the short channel effects in FD SOI MOSFETs. A similar concept was first proposed by M. Shur [1] by applying different gate bias in split-gate structure. But there was a problem of fringing capacitance which made it difficult to realize the two metal split-gate FET. As the distance between the two metal gates decreases, the fringing capacitance of the split-gate FET increases. The Dual Material Gate structure was first proposed by Long [2] in 1999. Gate material engineering was used instead of doping engineering [3-6]. When we are using gate material engineering, the channel potential is redistributed in such way that the short channel effects are reduced and transport of carriers is enhanced. There is a way of fabricating Hetro-Material Gate Structure by inserting one additional mask in the bulk CMOS processing technology which was first suggested by Zhou [7]. He also showed the characteristics of this new structure.

\section{SHORT CHANNEL EFFECTS}

Due to the extent of integration getting more and more, it is important to reduce the modern VLSI's power consumption. This power consumption may be reduced by using a lower power supply voltage. But, by doing so, the current driving capability will get degraded. Thus, there is a strong need of scaling MOS devices in order to improve the current driving capability so that we can fabricate VLSI chips with improved functionality [8]. If the channel length of a device is reduced, the threshold voltage of the device reduces. This dependence of device characteristics on channel length is a Short Channel Effect (SCE) due to which there is a variation in the device characteristics due to variation in the channel length during manufacturing. This also affects the controllability of the gate voltage over the channel and hence drain current. Thus, the subthreshold slope of the device gets degraded and there is an increase in the drain off-current as a result of increase in leakage. There are some popular ways of preventing SCEs like using shallow source/drain junctions and thinning gate oxide [13].

While scaling down the device, if the channel length is of the order of channel depth, then SCEs occur. When we scale down the device, channel length is reduced which reduces the threshold voltage and hence degrades the control of the gate voltage over the channel due to increase in the charge sharing effect from source/drain. The first SCE model proposed by Poon and Yau [9] describes the charge sharing effect by gate and drain electric fields in the depletion channel.

When the device is operating in the weak inversion mode, there exists a potential barrier between the source and the channel which the electrons have to cross before entering the channel. This barrier height should ideally be controlled by the gate voltage. But, due to DIBL (as indicated in Fig. 1), when we change the drain voltage, there is a change in this barrier height. If we increase the potential at the drain side, this barrier height gets reduced and more carriers enter the channel from source leading to an increase in the drain off-current of the device. Thus, due to DIBL, the drain voltage along with 
the gate voltage is responsible for controlling the drain current.

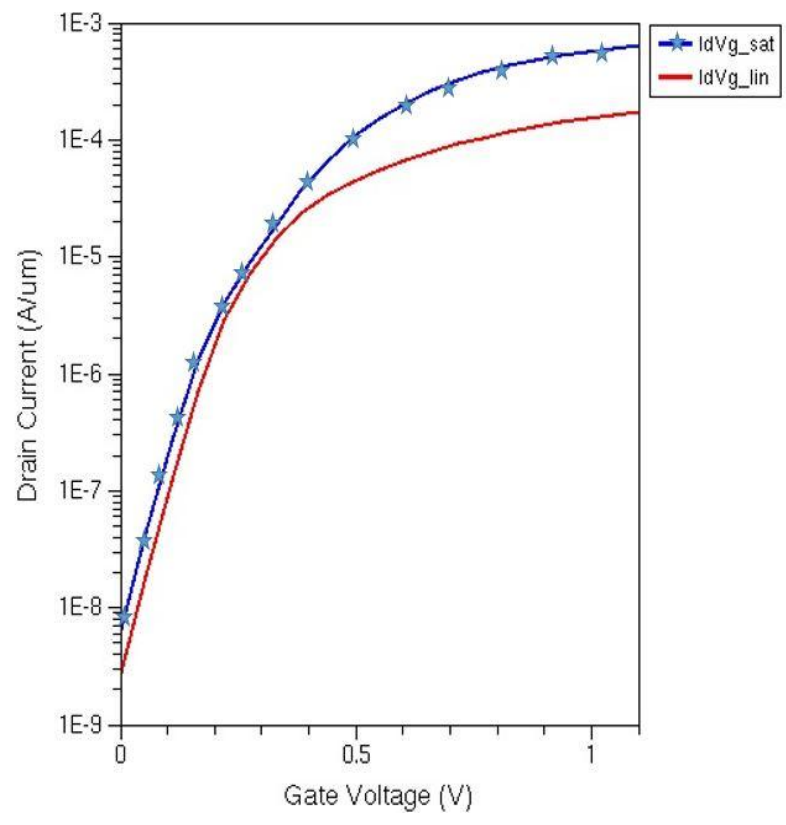

Fig 1: DIBL Curve of SMG FD SOI MOSFET

\section{DEVICE STRUCTURE}

The dual material gate FD SOI MOSFET structure is as shown in Fig. 2. As we are working at $25 \mathrm{~nm}$ technology, the channel length is $25 \mathrm{~nm}$, the lengths of source and drain are $45 \mathrm{~nm}$. Source/drain thickness is $6 \mathrm{~nm}$. The thickness of buried oxide layer is $20 \mathrm{~nm}$. Half of the gate is made up of Molybdenum and half of the gate is made up of Manganese.

One half of the gate near the source side is made up of Molybdenum and the other half of the gate is made up of Manganese. There is a reduction in hot carrier effect due to half of the gate at the drain side being made up of lower workfunction metal which reduces the peak electric field.

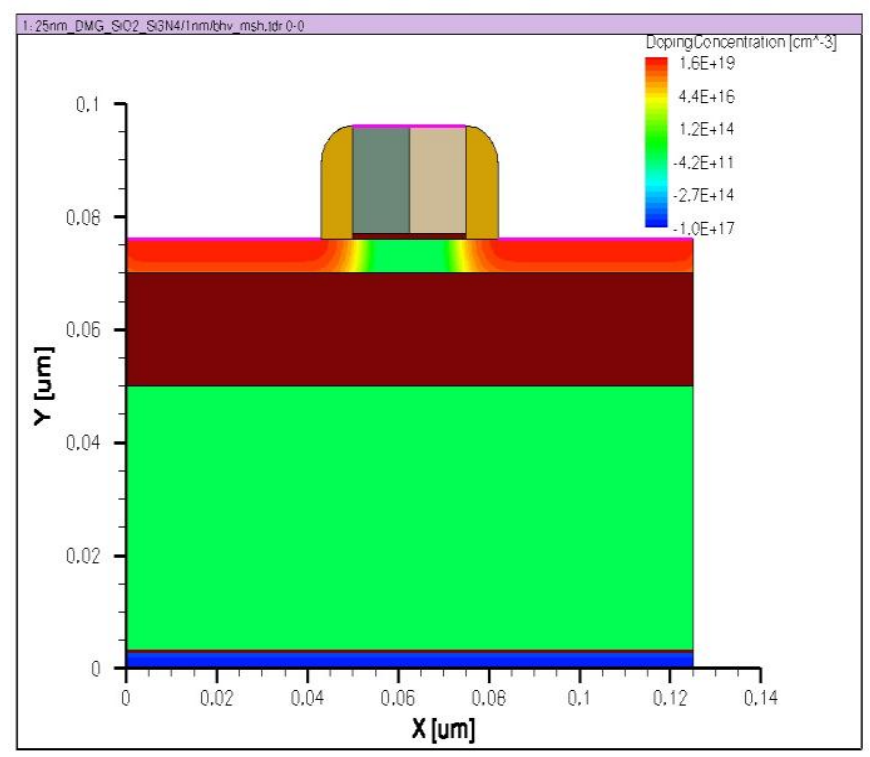

Fig 2: Device structure of DMG FD SOI MOSFET

Also, there is an increase in the average velocity of electrons due to the half of the gate at the source side being made up of higher work function metal which enhances the peak electric field at the source side. Thus, in the dual material gate structure, there is an increase in transconductance and reduction in drain conductance.

\section{RESULTS AND DISCUSSIONS}

The DIBL curves for Single Material Gate Fully Depleted Silicon on Insulator MOSFET and Dual Material Gate Fully Depleted Silicon on Insulator MOSFET are plotted and the improvement in DIBL can be clearly seen from Fig. 3.

As explained earlier, there is a reduction in DIBL from 30.7 $\mathrm{mV} / \mathrm{V}$ for SMG FD SOI MOSFET to $10.1 \mathrm{mV} / \mathrm{V}$ for DMG FD SOI MOSFET. We have also studied and shown the effects of variation of dielectric constant of spacer material on Ion/Ioff ratio (Fig. 4), DIBL (Fig. 5), Subthreshold Slope (Fig. 6). There is a reduction in off current of the device due to increase in the threshold voltage with an increase in dielectric constant of the spacer material which results better subthreshold slope and increased Ion/Ioff ratio.

\section{CONCLUSIONS}

It is concluded that there is a significant performance improvement in case of Dual Material Gate Fully Depleted Silicon on Insulator MOSFET as compared to its single material gate counterpart. Also, we observe that with an increase in the dielectric constant of the spacer material, there is a reduction in leakage in the device and hence the Ion/Ioff ratio is getting improved as the dielectric constant of the spacer material is increased. Also, the DIBL and Subthreshold slope is reduced with an increase in the dielectric constant of 
the spacer material. Thus, DMG MOSFET is suitable candidate for the VLSI application in nanometer regime.

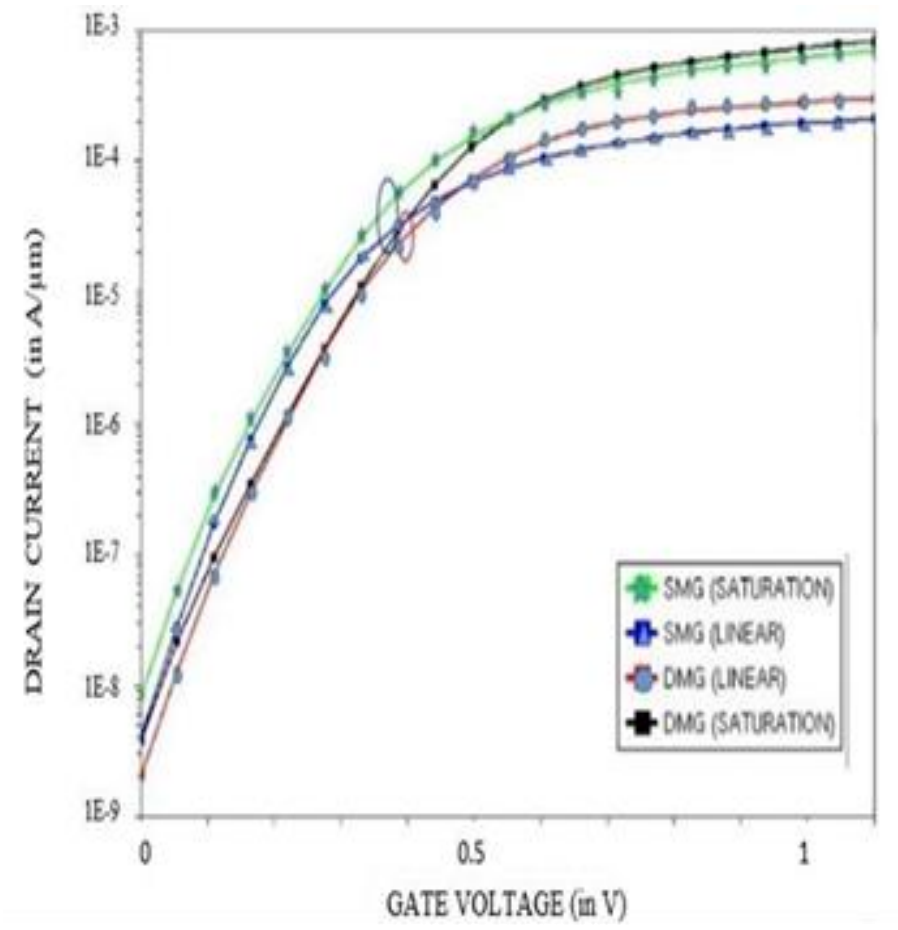

Fig 3: Comparison of DIBL Curves of SMG and DMG FD SOI MOSFET

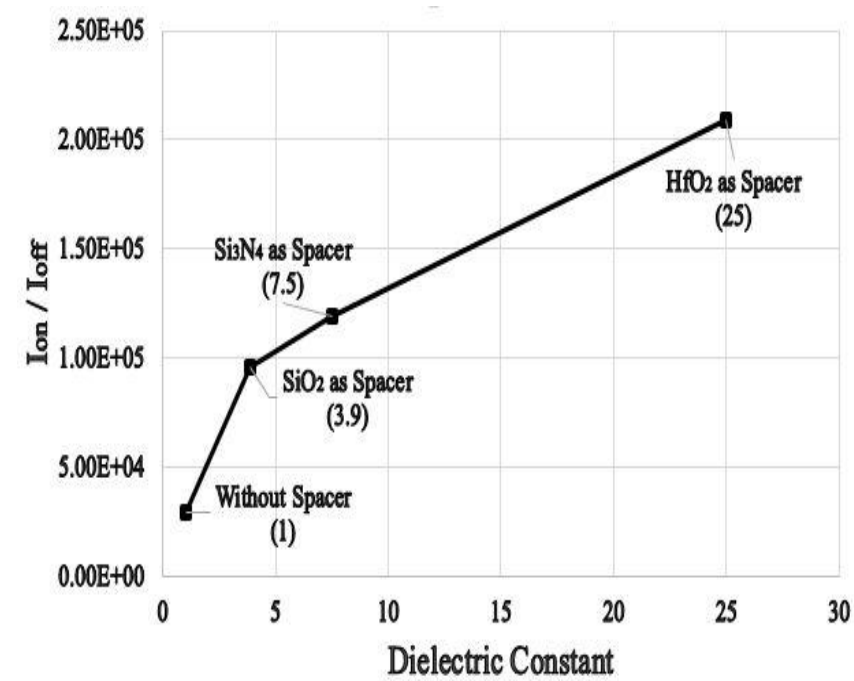

Fig 4: $\mathrm{I}_{\mathrm{on}} / \mathrm{I}_{\mathrm{off}}$ graph of DMG FD SOI MOSFET for different spacer materials

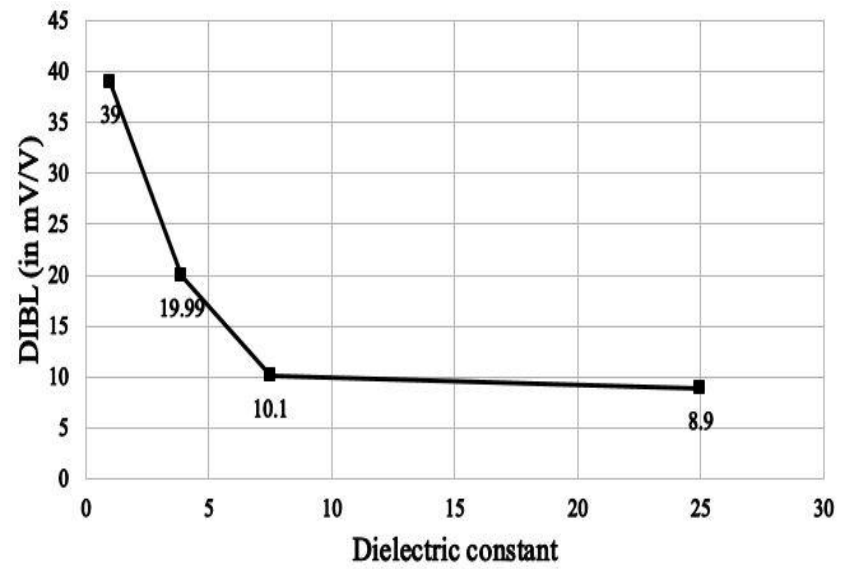

Fig 5: DIBL graph of DMG FD SOI MOSFET for different spacer materials

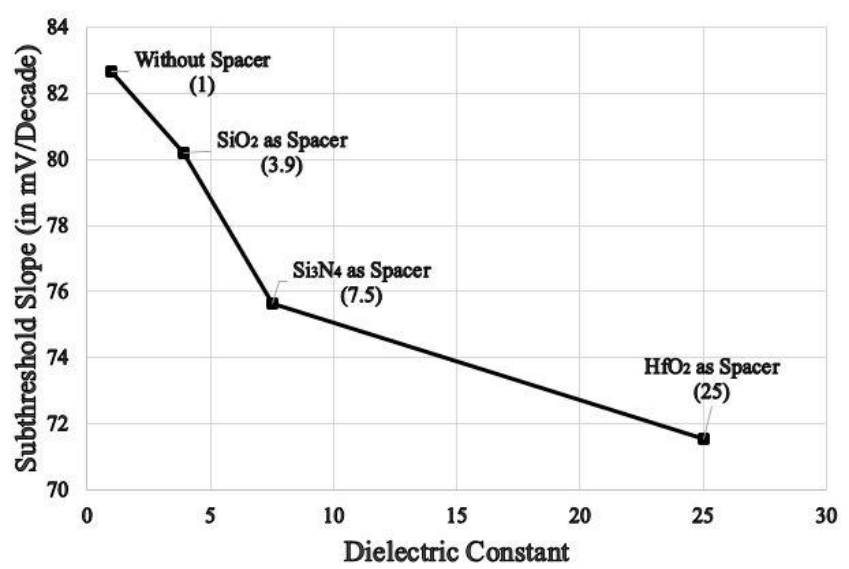

Fig 6: Subthreshold graph of DMG FD SOI MOSFET for different spacer materials

\section{REFERENCES}

[1]. M. Shur, "Split-gate field-effect transistor," Appl. Phys. Lett., vol. 54, no. 2, pp. 162-164, 1989.

[2]. W. Long, H. Ou, J.-M. Kuo, and K. K. Chin, "Dual material gate (DMG) Field Effect Transistor," IEEE Trans. Electron Devices, Vol. 46, pp. 865-870, May 1999.

[3]. A. Hiroki, S. Odanaka, and A. Hori, "A high performance $0.1 \mu \mathrm{m}$ MOSFET with asymmetric channel profile," in IEDM Tech. Dig., 1995, pp. 439-442

[4]. T. N. Buti, S. Ogura, N. Rovedo, K. Tobimatsu, and C. F. Codella, "Asymmetrical halo source GOLD drain (HS-GOLD) deep sub-half micron n-MOSFET design for reliability and performance," in IEDM Tech. Dig., 1989, pp. 617-620

[5]. T. Horiuchi, T. Homma, Y. Murao, and K. Okumura, "An asymmetric sidewall process for high performance LDD MOSFET's," IEEE Trans. Electron Devices, Vol. 41, pp. 186190, Feb. 1994.

[6]. J. F. Chen, J. Tao, P. Fang, and C. Hu, " $0.35-\mu \mathrm{m}$ asymmetric and symmetric LDD device comparison using a 
reliability/speed/power methodology," IEEE Electron Device Letters., Vol. 19, pp. 216-218, June 1998.

[7]. X. Zhou, "Exploring the novel characteristics of HeteroMaterial Gate Field-Effect transistors (HMGFET's) with gatematerial engineering," IEEE Trans. Electron Devices, Vol. 47, pp.113-120, January 2000.

[8]. T. Tsuchiya, Y. Sato, and M. Tomizawa, "Three Mechanisms Determining Short-Channel Effects in FullyDepleted SOI MOSFET's," IEEE Trans. Electron Devices, Vol. 45, pp 1116-1121, May 1998.

[9]. H. C. Poon, L. D. Yau, R. L. Johnston, and D. Beecham, "DC model for short-channel IGFET's," in IEDM Tech. Dig., pp. 156-159, 1974.

[10]. R. R. Troutman, "VLSI limitation from drain-induced barrier lowering," IEEE Trans. Electron Devices, vol. ED-26, pp. 461-469, April 1979.

[11]. M. J. Kumar and A. Chaudhry, "Two-dimensional analytical modelling of fully depleted dual-material gate (DMG) SOI MOSFET and evidence for diminished shortchannel effects," IEEE Trans. Electron Devices, vol. 51, pp. 569-574, Apr. 2004.

[12]. J. P. Colinge, "Hot electron effects in silicon-oninsulator n-channel MOSFETs," IEEE Trans. Electron Devices, vol. 34, pp. 2173-2177, 1987.

[13]. M. J. Kumar and A. Chaudhry, "Controlling ShortChannel Effects in Deep-Submicron SOI MOSFETs for Improved Reliability: A Review," IEEE Trans. Device And Materials Reliability, vol. 4, pp. 99-109, March 2004.

\section{BIOGRAPHIES}

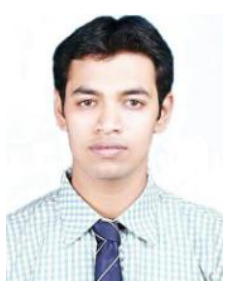

B.H.V SHRIKANT received the B.E degree in Electronics and Telecommunication Engineering from Shri Shankaracharya Institute of Technology and Management, Bhilai, INDIA in 2012. He is currently pursuing M.Tech degree at NIT Hamirpur, H.P, INDIA. His research interests include simulation of novel device structures on DMG SOI MOSFETs.

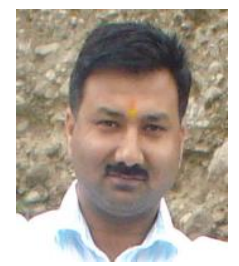

Ashwani K. Rana was born in Kangra, H.P., India in 1974. He received his B.Tech degree in Electronics and Communication Engineering from National Institute of Technology, Hamirpur, India and M.Tech degree in VLSI Technology from Indian Institute of Technology, Roorkee, India. He did his the Ph.D degree from National Institute of Technology, Hamirpur, India in the field of Nano Devices.

Presently he is with Department of Electronics and Communication Engineering, National Institute of Technology, Hamirpur, India, as an Assistant Professor.
His research interest include modeling of semiconductor devices, low power high performance VLSI circuit design and emerging integrated circuit technologies. He has guided one $\mathrm{Ph} . \mathrm{D}$ and guiding two more $\mathrm{Ph} . \mathrm{Ds}$ in these areas. He has more than 50 publications in International/National Journal \& conferences and guided more than $25 \mathrm{M}$.Tech students in these areas. He is member of ISTE 